\title{
Pneumococcal DNA is not detectable in the blood of healthy carrier children by real-time PCR targeting the lytA gene
}

Correspondence

Chiara Azzari

chiara.azzari@unifi.it

Received 23 November 2010

Accepted 18 February 2011

\author{
Chiara Azzari, Martina Cortimiglia, Maria Moriondo, Clementina Canessa, \\ Francesca Lippi, Federica Ghiori, Laura Becciolini, Maurizio de Martino \\ and Massimo Resti
}

Department of Paediatrics, Anna Meyer Children's University Hospital, Florence, Italy

\section{INTRODUCTION}

Streptococcus pneumoniae is the leading cause of invasive bacterial infections in children. Worldwide, an estimated 10.6 million children below the age of 5 years present with pneumococcal disease every year. Other at-risk populations include the elderly and immunosuppressed. Overall, more than 1.6 million deaths per year are attributable to such infections (Black et al., 2003; WHO, 2007). Establishing a microbiological diagnosis is important for proper treatment and epidemiological analysis.

Culture has been considered the gold standard for diagnosing invasive pneumococcal disease (IPD). However, this method suffers from a lack of sensitivity, especially when used after antibiotic therapy has been started to treat the infection (Azzari et al., 2010; Resti et al., 2009) or when small volumes are available, as is common in paediatric practice (Isaacman et al., 1996). An immunochromatographic method to detect bacterial cell-wall C polysaccharide antigen excreted in urine is sensitive but does not appear to distinguish between colonization and invasive

Abbreviations: $C_{t}$, threshold cycle; IPD, invasive pneumococcal disease. disease. Potentially, these obstacles could be overcome by the use of quantitative PCR.

Recently, it has been demonstrated that molecular methods for detecting bacterial DNA in blood or other normally sterile body fluids can be used for the efficient diagnosis and serotyping of IPD (Antonio et al., 2009; Azzari et al., 2008, 2010; Blaschke et al., 2010; Carvalho et al., 2010; Chiba et al., 2009; Corless et al., 2001; Saha et al., 2008; Strachan et al., 2011; Van Gastel et al., 2007). These methods do not require viable bacteria, need only small sample volumes and appear to be more sensitive than culture methods, making them potentially useful tools for the diagnosis and serotyping of pneumococcal pneumonia (Resti et al., 2010), even in conjunction with antibiotic treatment (Resti et al., 2009). A large number of clinical diagnostic laboratories around the world now use molecular methods as part of their routine testing. However, whenever a test appears to be more sensitive than those previously considered as gold standards, it is important to determine whether this increase in sensitivity is accompanied by a reduction in specificity and hence an increase in the rate of false-positives. For example, tests performed 
in the past using end-point PCR and primers targeting part of the streptococcal pneumolysin (ply) gene sequence reported finding positive results in the blood of healthy subjects even in the absence of $S$. pneumoniae colonization (Dagan et al., 1998; Murdoch et al., 2003).

The aim of the present study was to determine the frequency of positive PCR results in the blood of healthy carriers and non-carriers (i.e. false-positives) when assayed with a sensitive real-time PCR employing reagents to detect the pneumococcal autolysin $(l y t A)$ gene sequence, which is reported to be more specific than other genes such as ply (Abdeldaim et al., 2010; Carvalho et al., 2007).

\section{METHODS}

Subjects. The study included 147 healthy children between the ages of 6 months and 16 years (median 4.9 years, interquartile range 6.4 years), who were present at the Anna Meyer Children's Hospital, Florence, Italy, for routine examinations related to allergies or coeliac disease. None of the healthy subjects had taken antibiotics or received anti-pneumococcal vaccination for at least 1 month prior to the examination. Culture-positive biological samples of blood (20 samples) and cerebrospinal fluid (15 samples) were also tested. Moreover, real-time PCR was performed on 15 urine samples from patients with invasive pneumococcal infection (meningitis or pneumonia) whose blood samples were positive by real-time PCR.

PCR conditions. The presence of $S$. pneumoniae DNA in blood, urine or nasal swabs was evaluated by real-time PCR as described previously (Azzari et al., 2010). Primers and probes within the previously published lytA gene sequence (Carvalho et al., 2007) were used in PCR amplifications at a final concentration of $400 \mathrm{nM}$ for the primers and $400 \mathrm{nM}$ for the probe, which was labelled with 6-carboxy-4', $5^{\prime}$-dichloro-2', $7^{\prime}$-dimethoxyfluorescein (JOE). A negative control (no template) and a positive control were included in every PCR run. Total DNA was extracted from $200 \mu$ l blood or urine using a QIAmp DNeasy Blood \& Tissue kit (Qiagen), according to the manufacturer's instructions. Nasal swabs were placed in $1 \mathrm{ml}$ PBS and mixed by vortexing for $1 \mathrm{~min}$, and DNA was extracted from a $200 \mu \mathrm{l}$ sample. Real-time PCR was performed with an ABI 7000 sequence detection system (Applied Biosystems) using the following cycling parameters: $95{ }^{\circ} \mathrm{C}$ for $10 \mathrm{~min}$, followed by 45 cycles of $95{ }^{\circ} \mathrm{C}$ for $15 \mathrm{~s}$ and $60{ }^{\circ} \mathrm{C}$ for $1 \mathrm{~min}$. Cycle threshold $\left(C_{\mathrm{t}}\right)$ values were used to identify amplification of the target sequence and to estimate bacterial load. Samples were assumed to be negative if no increase in fluorescence signal was observed after 45 cycles. All reactions were performed in triplicate.

For quantitative analysis of carriage density, the amount of bacterial DNA was obtained by direct extrapolation of the $C_{\mathrm{t}}$ values to the amount of DNA (in copies $\mathrm{ml}^{-1}$ ) as read from the concentration versus $C_{\mathrm{t}}$ standard curve, as described previously (Rello et al., 2009; Resti et al., 2010).

S. pneumoniae bacterial cell-wall antigens were detected using a $\mathrm{C}$ polysaccharide antigen NOW Urinary Antigen Assay (Binax) according to the manufacturer's instructions.

Power of the study. Very few data are available on PCR positivity for S. pneumoniae in the blood of healthy children (Dagan et al., 1998; Murdoch et al., 2003).

A rate of false positivity in the diagnosis of invasive pneumococcal infection below $0.7 \%$ was considered acceptable in the clinical setting, being one-quarter of the false-positive samples obtained with culture methods, which, in a paediatric setting, appears to be around $2.8 \%$
(Sard et al., 2006). For that reason, as we hypothesized that we would not find a single positive with our method, the study was designed to include at least 142 healthy children, in the hope of finding at least one positive result that would allow quantification of the false-positive rate. For comparison of our method (real-time PCR of $l y t A$ ) with previous reports evaluating the presence of pneumococcal DNA in healthy children (nested end-point PCR of ply), assuming a carriage rate of $50 \%$ for the paediatric population studied, a sample size of only 80 subjects would be sufficient to detect a statistically significant difference.

\section{RESULTS AND DISCUSSION}

The low sensitivity of methods that require culture for the detection of pneumococcal disease may be due in part to their sensitivity to antibiotic therapy. PCR is a more sensitive assay (Antonio et al., 2009; Azzari et al., 2008, 2010; Corless et al., 2001; Saha et al., 2008; Van Gastel et al., 2007); however, the question of its specificity has been raised by the reported findings of positive PCR results from the blood of healthy subjects (Dagan et al., 1998; Murdoch et al., 2003). Both of these studies used sequences from the pneumococcal ply gene as the target for PCR. Recently, the specificity of the ply gene as a target for PCRbased detection of IPD has been questioned (Abdeldaim et al., 2009; Neeleman et al., 2004). When ply is used for diagnosis of pneumococcal infection on bronchoalveolar lavage or sputum samples, the presence in such samples of other bacteria such as Streptococcus mitis or Streptococcus oralis can be the cause of false-positive results, as these bacteria are known to contain similar sequences (Abdeldaim et al., 2009). However, it is difficult to imagine that a similar mechanism might be the cause of positivity for ply sequences in the blood of healthy subjects, and technical issues should also be considered. In fact, the studies that found positivity for ply sequences in the blood of healthy subjects used a nested end-point PCR, which is known to give a higher rate of false-positive results because it requires greater manipulation of the samples and amplicons. Recently, the lytA gene sequence has been suggested as more specific for the diagnosis of pneumococcal disease (Abdeldaim et al., 2010; Carvalho et al., 2007) and real-time PCR appears to be a more sensitive and specific tool for microbiological analysis. Therefore, we undertook the present study to determine the false-positive rate using real-time PCR with the pneumococcal lytA gene as the target.

We cannot exclude the possibility that carriage densities in the previous studies were higher than in our patients, thus affecting the passage of bacteria to the blood. However, the results of our study seem to contradict this hypothesis; in fact, the carriage density in the 77 carrier children in our study was in some subjects very high: values ranged between 1.6 and 5.2 $\log$ copies $\mathrm{ml}^{-1}$, with a mean value of $2.4 \log$ copies $\mathrm{ml}^{-1}$ and an interquartile range of 2.2-3.7 log copies $\mathrm{ml}^{-1}$.

\section{Carrier status}

In total, 77 of the 147 subjects $(52.4 \%)$ were positive for pneumococcal colonization of the pharynx using our 
real-time PCR method on nasal swabs. This is consistent with the expected $50 \%$ carriage rate for this population that we used in our power calculation. The age distribution of carrier status is presented in Fig. 1. There were few carriers among children under the age of 1 year and among children over the age of 13 years. This reflects the typical pattern of low carriage rates for very young children, followed by increasing rates that peak between 1 and 2 years of age and then decline, rates being much lower in adults (Antonio et al., 2009; Azzari \& Resti, 2008; Leino et al., 2001; Regev-Yochay et al., 2004).

\section{Pneumococcal DNA in blood}

Regardless of carrier status, we did not find positive results with PCR in the blood of any of the 147 subjects tested. The incidence of false-positive results appeared, therefore, to be equal to zero or at least less than $1 / 147(<0.7 \%)$ for children under the age of 16 years. PCR analysis of the culture-positive biological samples yielded positive results in $100 \%(15 / 15)$ of cerebrospinal fluid samples and $95 \%$ $(19 / 20)$ of blood samples, with an overall sensitivity of $97 \%(34 / 35)$. This is in agreement with our findings in a large population of children with pneumonia (Resti et al., 2010), where among the 292 patients who were tested with both culture and real-time PCR methods, S. pneumoniae was detected by one of the two methods in 47 patients, 45 of whom (96\%) were positive by real-time PCR with a sensitivity of real-time PCR that was four times higher than that of culture (Resti et al., 2010).

\section{Antigenuria}

We found that, despite having negative PCR results for pneumococcal DNA in the blood, 44 of the 77 carriers $(57.1 \%)$ had positive results for antigen in the urine, whilst the 70 non-carriers were all negative. This finding is consistent with previous reports of positive tests in a large percentage of children (Charkaluk et al., 2006; Domínguez et al., 2003; Dowell et al., 2001). In an attempt to understand this phenomenon, we examined urine samples for the presence of bacterial DNA and found none, indicating that colonization of the urinary tract was unlikely. We examined whether a correlation existed between the bacterial load colonizing the pharynx, as estimated by quantitative real-time PCR, and positivity for antigen in the urine, but did not find a significant correlation $(P=0.41)$. Moreover, there was no strong correlation between antigen in the urine and age $(P=0.09)$.

The absence of pneumococcal DNA in the blood and urine argues against disseminated infection as the source of antigen. We speculate that the antigenuria results from ingestion of colonized nasal secretions, followed by absorption of C-polysaccharide antigen from the digestive tract and excretion by the kidneys. Our findings serve to underline that, whilst useful for testing in adults where the carriage rate is low (Smith et al., 2009), tests for C-polysaccharide antigen in urine are not useful in children because a positive result can be due to either colonization or IPD. With regard to the presence of pneumococcal DNA in the urine of IPD patients, we demonstrated that only $2 / 15$ blood-positive IPD patients $(13.3 \%)$ also had positive urine samples. PCR performed on urine samples thus seems less sensitive than PCR or cultures performed on blood in patients with IPD. Larger studies designed to compare PCR sensitivity in different biological fluids are needed to confirm this point.

Real-time PCR assays of normally sterile body fluids, on the other hand, are specific for invasive disease in children and are more sensitive than culture methods. Using highly sensitive real-time PCR avoids the use of nested PCR

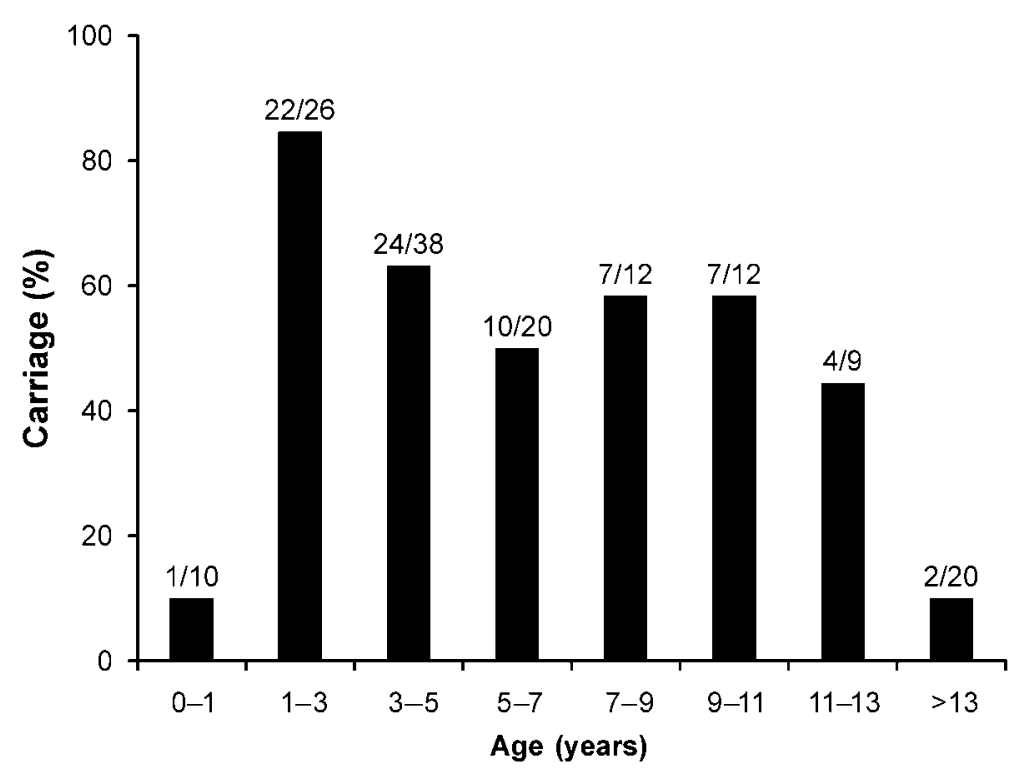

Fig. 1. S. pneumoniae carrier status according to age, as determined by real-time PCR analysis of nasal swabs. 
assays, which are associated with lower specificity (Avni et al., 2010) and present more possibilities for introducing method-based false-positives from contamination.

One limitation of the present study was that it was only performed on children with intact immune responses. Future studies will need to investigate the other two major at-risk populations - adults over the age of 65 and immunosuppressed subjects (Black et al., 2003) - to determine whether the method is also applicable to these populations.

In conclusion, real-time PCR detecting the lytA gene sequence is both sensitive and specific and can be a useful tool in the routine diagnosis of IPD. Its sensitivity, which surpasses that of any other method for this purpose, does not come at the cost of reduced specificity.

\section{REFERENCES}

Abdeldaim, G., Herrmann, B., Korsgaard, J., Olcén, P., Blomberg, J. \& Strålin, K. (2009). Is quantitative PCR for the pneumolysin (ply) gene useful for detection of pneumococcal lower respiratory tract infection? Clin Microbiol Infect 15, 565-570.

Abdeldaim, G., Herrmann, B., Mölling, P., Holmberg, H., Blomberg, J., Olcén, P. \& Strålin, K. (2010). Usefulness of real-time PCR for lytA, ply, and Spn9802 on plasma samples for the diagnosis of pneumococcal pneumonia. Clin Microbiol Infect 16, 1135-1141.

Antonio, M., Hakeem, I., Sankareh, K., Cheung, Y. B. \& Adegbola, R. A. (2009). Evaluation of sequential multiplex PCR for direct detection of multiple serotypes of Streptococcus pneumoniae from nasopharyngeal secretions. J Med Microbiol 58, 296-302.

Avni, T., Mansur, N., Leibovici, L. \& Paul, M. (2010). PCR using blood for diagnosis of invasive pneumococcal disease: systematic review and meta-analysis. J Clin Microbiol 48, 489-496.

Azzari, C. \& Resti, M. (2008). Reduction of carriage and transmission of Streptococcus pneumoniae: the beneficial "side effect" of pneumococcal conjugate vaccine. Clin Infect Dis 47, 997-999.

Azzari, C., Moriondo, M., Indolfi, G., Massai, C., Becciolini, L., de Martino, M. \& Resti, M. (2008). Molecular detection methods and serotyping performed directly on clinical samples improve diagnostic sensitivity and reveal increased incidence of invasive disease by Streptococcus pneumoniae in Italian children. J Med Microbiol 57, 1205-1212.

Azzari, C., Moriondo, M., Indolfi, G., Cortimiglia, M., Canessa, C., Becciolini, L., Lippi, F., de Martino, M. \& Resti, M. (2010). Realtime PCR is more sensitive than multiplex PCR for diagnosis and serotyping in children with culture negative pneumococcal invasive disease. PLoS ONE 5, e9282.

Black, R. E., Morris, S. S. \& Bryce, J. (2003). Where and why are 10 million children dying every year? Lancet 361, 2226-2234.

Blaschke, A. J., Heyrend, C., Byington, C. L., Obando, I., VazquezBarba, I., Doby, E. H., Korgenski, E. K., Sheng, X., Poritz, M. A. \& other authors (2010). Molecular analysis improves pathogen identification and epidemiologic study of pediatric parapneumonic empyema. Pediatr Infect Dis J (Epub ahead of print: doi:10.1097/ INF.0b013e3182002d14)

Carvalho, M. G., Tondella, M. L., McCaustland, K., Weidlich, L., McGee, L., Mayer, L. W., Steigerwalt, A., Whaley, M., Facklam, R. R. \& other authors (2007). Evaluation and improvement of real-time PCR assays targeting lytA, ply, and psaA genes for detection of pneumococcal DNA. J Clin Microbiol 45, 2460-2466.
Carvalho, M. G., Pimenta, F. C., Jackson, D., Roundtree, A., Ahmad, Y., Millar, E. V., O'Brien, K. L., Whitney, C. G., Cohen, A. L. \& Beall, B. W. (2010). Revisiting pneumococcal carriage by use of broth enrichment and PCR techniques for enhanced detection of carriage and serotypes. J Clin Microbiol 48, 1611-1618.

Charkaluk, M. L., Kalach, N., Mvogo, H., Dehecq, E., Magentie, H., Raymond, J., Gendrel, D., Kremp, O. \& Decoster, A. (2006). Assessment of a rapid urinary antigen detection by an immunochromatographic test for diagnosis of pneumococcal infection in children. Diagn Microbiol Infect Dis 55, 89-94.

Chiba, N., Murayama, S. Y., Morozumi, M., Nakayama, E., Okada, T., Iwata, S., Sunakawa, K. \& Ubukata, K. (2009). Rapid detection of eight causative pathogens for the diagnosis of bacterial meningitis by real-time PCR. J Infect Chemother 15, 92-98.

Corless, C. E., Guiver, M., Borrow, R., Edwards-Jones, V., Fox, A. J. \& Kaczmarski, E. B. (2001). Simultaneous detection of Neisseria meningitidis, Haemophilus influenzae, and Streptococcus pneumoniae in suspected cases of meningitis and septicemia using real-time PCR. J Clin Microbiol 39, 1553-1558.

Dagan, R., Shriker, O., Hazan, I., Leibovitz, E., Greenberg, D., Schlaeffer, F. \& Levy, R. (1998). Prospective study to determine clinical relevance of detection of pneumococcal DNA in sera of children by PCR. J Clin Microbiol 36, 669-673.

Dominguez, J., Blanco, S., Rodrigo, C., Azuara, M., Gali, N., Mainou, A., Esteve, A., Castellvi, A., Prat, C. \& other authors (2003). Usefulness of urinary antigen detection by an immunochromatographic test for diagnosis of pneumococcal pneumonia in children. J Clin Microbiol 41, 2161-2163.

Dowell, S. F., Garman, R. L., Liu, G., Levine, O. S. \& Yang, Y. H. (2001). Evaluation of Binax NOW, an assay for the detection of pneumococcal antigen in urine samples, performed among pediatric patients. Clin Infect Dis 32, 824-825.

Isaacman, D. J., Karasic, R. B., Reynolds, E. A. \& Kost, S. I. (1996). Effect of number of blood cultures and volume of blood on detection of bacteremia in children. J Pediatr 128, 190-195.

Leino, T., Auranen, K., Jokinen, J., Leinonen, M., Tervonen, P. \& Takala, A. K. (2001). Pneumococcal carriage in children during their first two years: important role of family exposure. Pediatr Infect Dis J 20, 1022-1027.

Murdoch, D. R., Anderson, T. P., Beynon, K. A., Chua, A., Fleming, A. M., Laing, R. T., Town, G. I., Mills, G. D., Chambers, S. T. \& Jennings, L. C. (2003). Evaluation of a PCR assay for detection of Streptococcus pneumoniae in respiratory and nonrespiratory samples from adults with community-acquired pneumonia. J Clin Microbiol 41, 63-66.

Neeleman, C., Klaassen, C. H., Klomberg, D. M., de Valk, H. A. \& Mouton, J. W. (2004). Pneumolysin is a key factor in misidentification of macrolide-resistant Streptococcus pneumoniae and is a putative virulence factor of S. mitis and other streptococci. J Clin Microbiol 42, 4355-4357.

Regev-Yochay, G., Raz, M., Dagan, R., Porat, N., Shainberg, B., Pinco, E., Keller, N. \& Rubinstein, E. (2004). Nasopharyngeal carriage of Streptococcus pneumoniae by adults and children in community and family settings. Clin Infect Dis 38, 632-639.

Rello, J., Lisboa, T., Lujan, M., Gallego, M., Kee, C., Kay, I., Lopez, D., Waterer, G. W. \& DNA-Neumococo Study Group (2009). Severity of pneumococcal pneumonia associated with genomic bacterial load. Chest 136, 832-840.

Resti, M., Micheli, A., Moriondo, M., Becciolini, L., Cortimiglia, M., Canessa, C., Indolfi, G., Bartolini, E., de Martino, M. \& Azzari, C. (2009). Comparison of the effect of antibiotic treatment on the possibility of diagnosing invasive pneumococcal disease by culture or molecular methods: a prospective, observational study of children 
and adolescents with proven pneumococcal infection. Clin Ther 31, 1266-1273.

Resti, M., Moriondo, M., Cortimiglia, M., Indolfi, G., Canessa, C., Becciolini, L., Bartolini, E., Benedictis, F. M., de Martino, M. \& other authors (2010). Community-acquired bacteremic pneumococcal pneumonia in children: diagnosis and serotyping by real-time polymerase chain reaction using blood samples. Clin Infect Dis 51, 1042-1049.

Saha, S. K., Darmstadt, G. L., Baqui, A. H., Hossain, B., Islam, M., Foster, D., Al-Emran, H., Naheed, A., Arifeen, S. E. \& other authors (2008). Identification of serotype in culture negative pneumococcal meningitis using sequential multiplex PCR: implication for surveillance and vaccine design. PLoS ONE 3, e3576.

Sard, B., Bailey, M. C. \& Vinci, R. (2006). An analysis of pediatric blood cultures in the postpneumococcal conjugate vaccine era in a community hospital emergency department. Pediatr Emerg Care 22, 295-300.
Smith, M. D., Sheppard, C. L., Hogan, A., Harrison, T. G., Dance, D. A., Derrington, P., George, R. C. \& South West Pneumococcus Study Group (2009). Diagnosis of Streptococcus pneumoniae infections in adults with bacteremia and community-acquired pneumonia: clinical comparison of pneumococcal PCR and urinary antigen detection. J Clin Microbiol 47, 1046-1049.

Strachan, R. E., Cornelius, A., Gilbert, G. L., Gulliver, T., Martin, A., McDonald, T., Nixon, G. M., Roseby, R., Ranganathan, S. \& other authors (2011). A bedside assay to detect Streptococcus pneumoniae in children with empyema. Pediatr Pulmonol 46, 179-183.

Van Gastel, E., Bruynseels, P., Verstrepen, W. \& Mertens, A. (2007). Evaluation of a real-time polymerase chain reaction assay for the diagnosis of pneumococcal and meningococcal meningitis in a tertiary care hospital. Eur J Clin Microbiol Infect Dis 26, 651-653.

WHO (2007). Pneumococcal conjugate vaccine for childhood immunization - WHO position paper. Wkly Epidemiol Rec 82, 93-104. 